\title{
Aportes para el abordaje interdisciplinar de la adherencia al tratamiento
}

\section{Treatment adherence: contributions to interdisciplinary approach}

\author{
Marco Vargas, Carolina Herrera, Lilia Rocha • Duitama (Colombia)
}

La adherencia al tratamiento (AT) es un tema fundamental para la salud de la población y para los profesionales que trabajan en atención de salud. Para la Organización Mundial de la Salud (OMS) (1), la adherencia deficiente al tratamiento es un alarmante problema mundial que cobra especial importancia en el ámbito de las enfermedades crónicas y de manera particular en las no transmisibles (ECNT), es decir aquellas que según la OMS (p4) (1), cuentan "con una o varias de las siguientes características: son permanentes, dejan discapacidad residual, son causadas por una alteración anatomopatológica irreversible, requieren adiestramiento especial del paciente para la rehabilitación o cabe prever que requieran un periodo largo de supervisión, observación o atención". A la falta de AT se le atribuyen altos índices de mortalidad prematura y pérdida de calidad de vida del paciente (2). De igual manera, hay consecuencias económicas por el alto impacto del costo en los sistemas de salud y en las personas (3). En su reflexión sobre el tema Silva, Galeano y Correa (4) apuntan que el fenómeno de la no adherencia se ha mantenido en una preocupante proporción de 59\% durante décadas, y además existen entre 33-69\% de hospitalizaciones secundarias al incumplimiento con el tratamiento. De ahí que se afirme que "mejorar la adherencia terapéutica quizá sea la mejor inversión para abordar efectivamente los procesos crónicos"(1) (p22).

El concepto de AT ha recibido numerosos nombres y se han propuesto distintas definiciones. Osterberg y Blaschke (5) la definieron como la medida en que el paciente toma la medicación indicada por los proveedores de cuidados de salud. El concepto restrictivo de adherencia como cumplimiento, centrado en la parte farmacológica de los tratamientos y adjudicando al paciente un papel pasivo, ha cambiado hacia propuestas como la de Martín, Grau y Espinosa (6) (p 289) quienes lo entienden como "un comportamiento complejo con carácter de proceso conformado por una estructura y dinámica interna, que integra un componente personal, un componente relacional en el que se encuentra implicado el profesional de salud y uno comportamental propiamente dicho, dirigidos al logro de un resultado beneficioso para la salud".

A la luz de los nuevos conceptos la AT es un comportamiento humano, modulado por distintos componentes subjetivos y objetivos, contextuales, estructurales y coyunturales, por lo que se puede asumir como: el comportamiento activo, automotivado y autodirigido del paciente, que implica el compromiso consigo mismo y con los profesionales de la salud que lo atienden, con el fin de alcanzar los objetivos biomédicos relacionados con el tratamiento acordado, la adopción de un estilo de vida más saludable, y el mejoramiento de su calidad de vida en consonancia con su proyecto vital. Es una compleja interacción entre componentes biológicos, conductuales, cognoscitivos y emocionales, que lleva a un alto grado de seguimiento del tratamiento (7). Esto implica la necesidad de formas de abordaje más complejas e integrales que sean consonantes con la dinámica biopsicosocial del ser humano.

Los factores que se han asociados con la AT constituyen una extensa serie (8-10). La OMS (1) los agrupa en cuatro áreas: relación médico-paciente; el paciente y su entorno; la enfermedad y la terapéutica. En una investigación con pacientes hipertensos (8) se identificaron como factores asociados al adecuado cumplimiento: la organización de los
Dr. Marco Orlando Vargas Olano: Médico. Psicólogo. Atención Primaria y Urgencias, Clínica Boyacá; Dra. Carolina Herrera Delgado: Psicóloga. UNAD. Magister Psicología; Dra. Lilia Mercedes Rocha-Nieto: Psicóloga. Universidad El Bosque. Magister Psicología. Duitama (Colombia).

Correspondencia: Dr. Marco Orlando Vargas Olano. Duitama (Colombia).

E-mail: movoco@gmail.com

Recibido: 29/05/016 Aceptado: 20/IV/2017 
servicios de salud, rasgos de personalidad, características del tratamiento, la percepción de gravedad y vulnerabilidad percibida de parte del paciente, la disponibilidad de apoyo familiar.

Delgado, Figueroa y Herrera (11) en su revisión relacionada con rehabilitación cardiaca los agrupan según tres niveles. Los relacionados con el paciente o nivel individual representan variables biomédicas, psicológicas y algunas socioeconómicas. Incluyen características de la enfermedad y complejidad del régimen terapéutico; conocimiento de la enfermedad, creencias y percepciones de la misma; el valor que el paciente asigna a su condición de salud y al tratamiento de su enfermedad; la autoeficacia o creencia en los beneficios de lo que se le prescribe o indica hacer y en su propia capacidad para ejecutarlo; tipo de estrategias de afrontamiento que utiliza ante la adversidad y los retos; estados afectivos negativos con presencia de estrés, angustia, ansiedad, depresión, desesperanza y frustración; cualidades como motivación, perseverancia, creatividad, tolerancia, memoria, atención; recursos económicos. El nivel microsocial está representado por factores como: redes de apoyo social, relación médico (equipo de salud) - paciente, ambiente en que vive el paciente o la ubicación geográfica. Finalmente están los factores de orden macrosocial como el sistema de salud con sus beneficios y limitaciones, las políticas gubernamentales frente al servicio y su calidad o el sistema socioeconómico en que se inserta, que son potentes determinantes de las posibilidades de acceso a servicios de salud y favorecen o entorpecen la AT.

Las conductas de AT hacen referencia a los que se pueden considerar los pilares de los tratamientos de las ECNT: régimen farmacológico, alimentación saludable, actividad física, eliminación de hábitos nocivos y vivencia adecuada de las emociones. El adecuado cumplimiento de cualquier plan terapéutico exige realizar una serie de tareas que requieren saber qué hacer, cómo hacer y cuándo hacer, así como disponer de varias habilidades para mantener a lo largo del tiempo todas las acciones que componen su programa y sus objetivos terapéuticos. Una idea de lo anterior se ofrece en la Tabla 1.

Los pacientes adherentes al tratamiento y al cuidado de su enfermedad deben efectuar constantemente acciones y tareas para alcanzar unas metas y mantenerse en ellas. Estos esfuerzos continuados de las personas orientados por un propósito, constituyen según Bandura (12) la autorregulación. Para Karoly (13) autorregulación se refiere a “... aquellos procesos, internos y/o transaccionales, que permiten a una persona guiar sus actividades hacia unas metas a través del tiempo y de las nuevas circunstancias (contextos). Implica modulación del pensamiento, el afecto, la conducta o la atención mediante el uso deliberado o automático de mecanismos específicos de apoyo y metahabilidades. En su ejercicio existen cinco fases interrelacionadas: 1) selección de objetivo, 2) conocimiento del objetivo; que comprende los conocimiento y actitudes acerca del problema de salud y de los comportamientos requeridos para superarlo o contenerlo; las creencias sobre la gravedad de la patología y la vulnerabilidad ante ella; 3) mantenimiento de la conducta en direccion al objetivo; 4) cambio de dirección o de nuevas prioridades, y 5) logro de meta". (p. 25).

Este proceso se afirma en motivación intrínseca, autogenerada (1) y lo integran tres subfunciones (12): automonitoreo de la conducta; auto evaluación de la conducta a la luz de normas personales, estándares sociales y circunstancias ambientales; y las reacciones afectivas y cognitivas, relacionada con el valor dado a las conductas saludables.

Desde la psicofisiología es una función superior, controlada por la corteza prefrontal. Implica la coordinación y manejo de estados cognoscitivos, conductuales y fisiológicos para desarrollar funciones como: planeación (fijar metas y seleccionar acciones y tareas para su cumplimiento en el tiempo mediato); monitoreo que permita detectar y controlar las discrepancias entre la conducta actual y los objetivos así como identificar tentaciones y controlarlas; juicio para evaluar y confrontar el valor dado a la propia condición de salud y a los objetivos terapeúticos contra los costos y

Tabla 1. Tareas que debe llevar a cabo un paciente afectado por algunas de las ECNT.

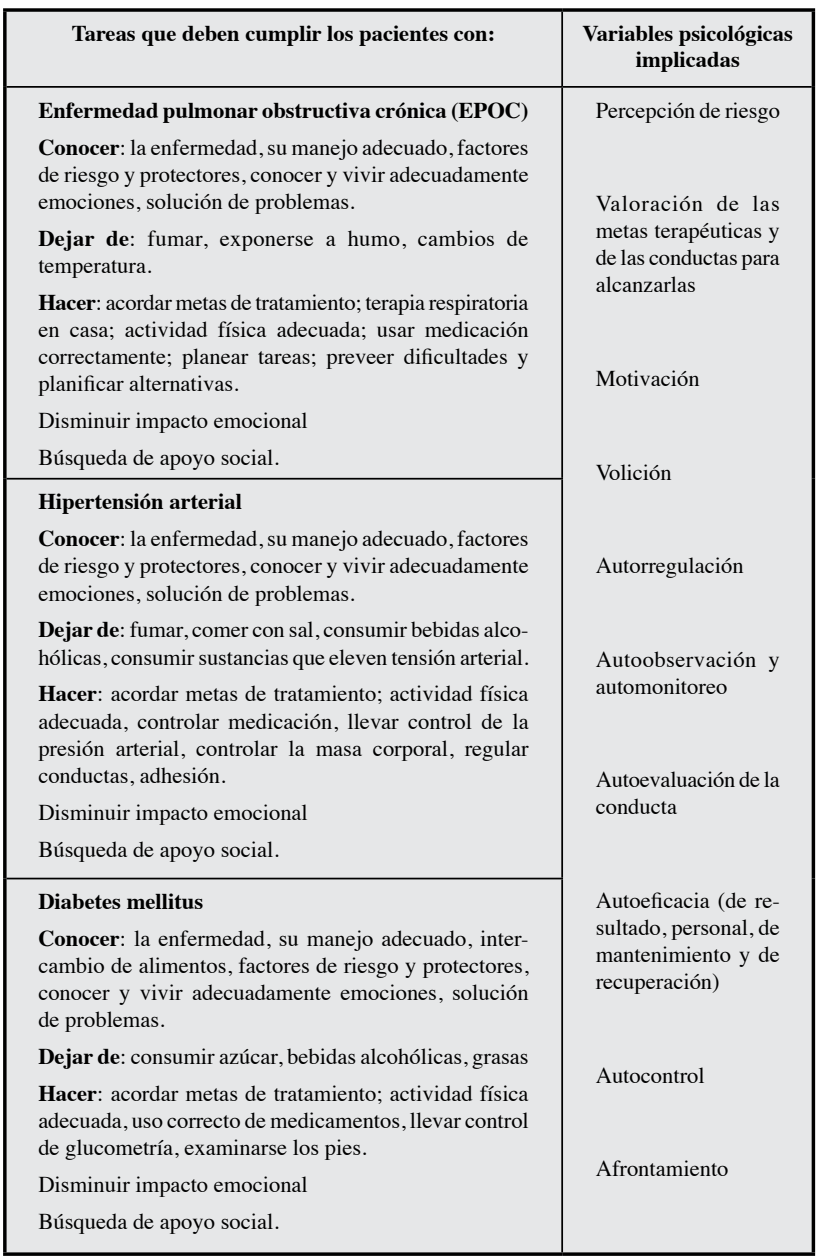


esfuerzos que el mismo demanda; autoobservación para detectar problemas. Por supuesto todo ello exige atención, concentración y perseverancia (14-18). Además de lo anterior mantener una conducta saludable demanda además adecuada información de retorno, refuerzo positivo (19-21).

Por otra parte las estrategias de autorregulación requieren la activación de un proceso de autocontrol (22).

Respecto al autocontrol, Rachlin (23) basado en el análisis experimental de la conducta, considera que es la elección de una alternativa que implica un beneficio mejor y mayor pero en el futuro, sobre otra que representa una satisfacción (no siempre un bien) inmediata. Combina variables como: esfuerzo cognoscitivo para concentrarse, identificar tentaciones, monitorear el propio comportamiento y entender las consecuencias del mismo; evaluar sus consecuencias a largo plazo con aplazamiento de la gratificación y refuerzo positivo de las conductas que se toman (24). Puede fallar en situaciones en las que los recursos personales son limitados (p. ej. el estrés o la falta de atención), en ciertas situaciones sociales (p. ej. compañía e influencia de los amigos), o cuando se trata de rutinas habituales muy consolidadas (20).

La autorregulación también abarca el mecanismo de autoeficacia (12) que es la confianza en el tratamiento y en las acciones que el sujeto realiza para llevarlo a cabo, así como en la propia capacidad para realizarlas, para perseverar en ellas a lo largo del tiempo y para ser capaz de retomarlas cuando hay recaídas (por ejemplo abandono temporal o parcial del tratamiento). En su desarrollo son fundamentales los actores sociales, incluyendo los equipos de salud. La autoeficacia influye en las aspiraciones y en las decisiones que se toman, impacta fuertemente el pensamiento, el afecto, la motivación y el ejercicio de la acción personal, modulando la cantidad de esfuerzo y de tiempo que se invierte en una tarea y la perseverancia ante las dificultades.

El entrenamiento en habilidades específicas y en estrategias apropiadas para afrontar y resolver problemas, promueve esta condición psicológica.

En síntesis, la realización constante de conductas protectoras de salud y la prevención de conductas de riesgo para la salud exige casi siempre un esfuerzo de autorregulación por el cual se establecen unas metas, se planean cursos de acción para lograrlas y se anticipan dificultades. Se trata de un constructo multidimensional como lo esquematiza la Figura 1 (25-29).

La psicología ha propuesto modelos que buscan explicar la modificación del comportamiento en cuanto a conductas de salud se trata $(30,31)$. Uno de ellos es el modelo procesual de acción en salud (HAPA, del inglés Health Action Process Approach) según el cual la adopción, iniciación y mantenimiento de los comportamientos de salud es un proceso con dos fases, una de motivación y otra de volición. La motivacional incluye tres variables que influyen en la intención de adoptar un comportamiento: percepción de riesgo, expectativas de resultado y autoeficacia. La volitiva o proceso de pasar de la intención a la acción, involucra cogniciones relacionadas con la iniciación y el control de la acción, y se subdivide en planeamiento, acción y mantenimiento. La contribución principal del modelo HAPA es la inclusión de dos factores volitivos mayores: autoeficacia volitiva (de mantenimiento del comportamiento y de recuperación de recaídas) y planificación estratégica (de las acciones y del afrontamiento) (20, 21, 25). Este modelo posee validaciones empíricas en distintos tipos de conductas saludables incluyendo las fases no supervisadas médicamente, que avalan su poder para explicar e inducir cambios conductuales $(25,28,32)$.

Las características tanto del constructo psicológico autorregulación, como del modelo HAPA, permiten proponer que la intervención sobre esta variable psicológica y la utilización del modelo son una vía plausible para abordar el complejo problema de la AT. En concreto los equipos interdisciplinares de salud (médicos, enfermeras, terapeuta físico, terapeuta respiratorio, nutricionista, psicólogo, trabajador social) actuando coordinada y cooperativamente deben fortalecer principalmente la autoeficacia y la planeación, para incidir positivamente en el incremento de la AT $(7,33)$.

El conocimiento y comprensión de estos aportes por parte de los integrantes de los equipos de atención en salud, facilitan su misión de ayuda a los pacientes para que ellos logren niveles adecuados de AT. Esto significa centrarse

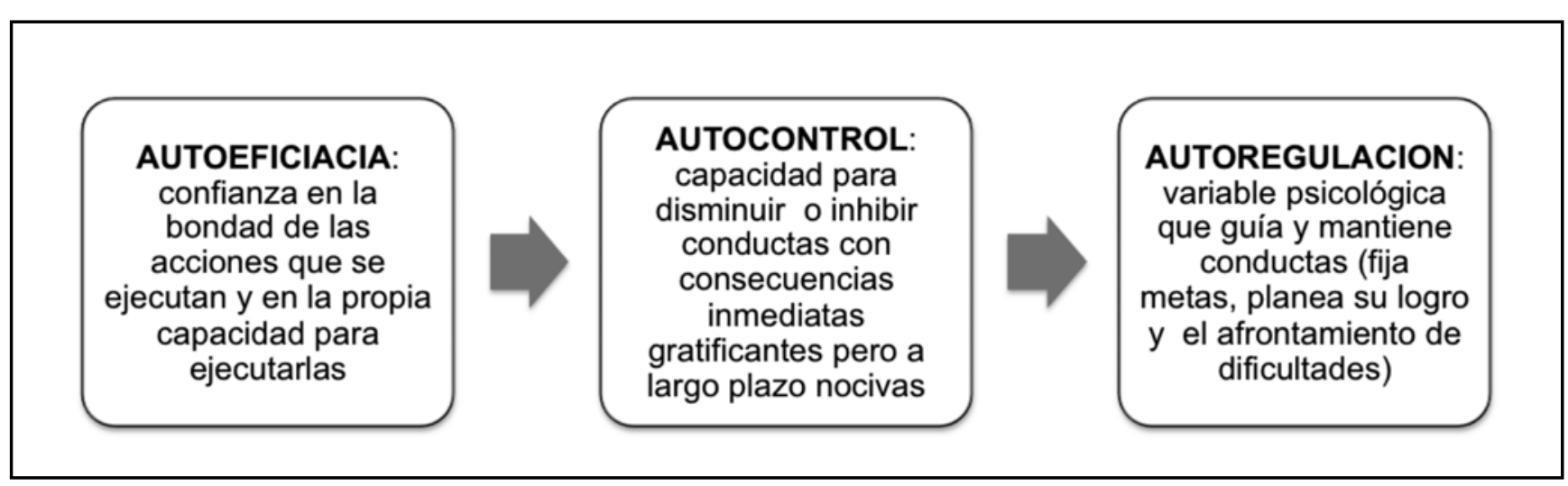

Figura 1. El complejo autorregulatorio. 


\begin{tabular}{|c|c|c|c|c|}
\hline \multirow[t]{2}{*}{ Momento o fase según Hapa } & \multicolumn{2}{|c|}{ Motivacional } & \multicolumn{2}{|c|}{ Acción } \\
\hline & Prevolitiva & Interfaz intención - acción & Mantenimiento & Recuperación \\
\hline Tareas del paciente & $\begin{array}{l}\text { Percibir vulnerabilidad . } \\
\text { Incrementar autoeficacia. } \\
\text { Confiar en eficacia de } \\
\text { resultados. }\end{array}$ & $\begin{array}{l}\text { Fijar metas. } \\
\text { Planear acción. } \\
\text { Planear afrontamiento de } \\
\text { dificultades. }\end{array}$ & Planear acciones cotidianas & $\begin{array}{l}\text { Planear afrontamiento de } \\
\text { recaídas }\end{array}$ \\
\hline Tarea equipo salud & $\begin{array}{l}\text { Conocer de la manera más } \\
\text { amplia posible características } \\
\text { individuales y condiciones } \\
\text { ambientales del paciente. } \\
\text { Ilustrar sobre enfermedad y } \\
\text { tratamiento. } \\
\text { Proveer parámetros de } \\
\text { comparación (normas o } \\
\text { valores normales). }\end{array}$ & $\begin{array}{l}\text { Posibilitar acuerdo con el } \\
\text { paciente sobre metas del } \\
\text { tratamiento. } \\
\text { Entrenar en habilidades } \\
\text { requeridas para cada plan } \\
\text { terapéutico. } \\
\text { Identificar necesidades y } \\
\text { recursos. }\end{array}$ & $\begin{array}{l}\text { Proveer información de } \\
\text { retorno. } \\
\text { Educación y entrenamiento } \\
\text { continuo a paciente. } \\
\text { Consolidar red de apoyo } \\
\text { social. } \\
\text { Ofrecer reforzamiento y } \\
\text { recompensa }\end{array}$ & $\begin{array}{l}\text { Proveer información de } \\
\text { retorno. } \\
\text { Educación y entrenamiento } \\
\text { continuo a pacientes. } \\
\text { Entrenar en resolución de } \\
\text { problemas. } \\
\text { Optimizar estrategias de } \\
\text { afrontamiento. } \\
\text { Consolidar red de apoyo } \\
\text { social. } \\
\text { Ofrecer reforzamiento y } \\
\text { recompensa }\end{array}$ \\
\hline
\end{tabular}

en el paciente, desarrollar habilidades de escucha activa, buscar un entendimiento cabal de las diferentes condiciones individuales y ambientales de cada persona para proveer la información adecuada, clara y completa para cada quien, así como entrenarlos en las habilidades concretas requeridas para llevar a cabo tratamientos que suelen ser complejos y multidimensionales. A esto se suma la búsqueda y obtención de apoyo social.

Una intervención de este tipo difícilmente estará al alcance de un profesional aislado, por lo que exige el desarrollo de un verdadero trabajo interdisciplinar.

Las posibles tareas del equipo de atención en salud, se ilustran en la Tabla 2

\section{Referencias}

1. Sabaté, E. Adherencia a los tratamientos a largo plazo. Pruebas para la acción. [Monografía en Internet]. Organización Mundial de la Salud. 2004. Consultado 1 octubre de 2014. Disponible en: http://www.paho.org/spanish/ad/dpc/nc/adhere ncia-largo-plazo.pdf.

2. Martin L, Grau J. La investigación de la adherencia terapéutica como un problema de la psicología de la salud. Psicología y salud. Ene - jul 2004;14(1):89-99.

3. Organización Mundial de la Salud. Prevención de las enfermedades crónicas una inversión vital. [Monografía en Internet]. OMS. 2003. Consultado 1 octubre 2014. Disponible en: http//www.who.int/chp/chronicdiseasereport/over view sp.pdf.

4. Silva GE, Galeano E, Correa JO. Adherencia al tratamiento. Implicaciones de la no-adherencia. Acta Med Colomb. Dic 2005 (Consultado: 8 de enero de 2012); 30(4):268-273. Disponible en: http://uaemex.redalyc.org/articulo. oa?id=163113820004.

5. Osterberg L, Blaschke T. Adherence to Medication. $N$ Engl J Med. Aug 2005;353(5):487-497.

6. Martín LA, Grau JA, Espinosa AD. Marco conceptual para la evaluación y mejora de la adherencia a los tratamientos médicos en enfermedades crónicas. Revista cubana de salud pública. [revista en Internet]. 2014 (Consultada 01 de octubre 2014);(40)2:222-238. Disponible en: http://www.scielosp.org/scielo. php?script=sci_arttext\&pid=S0864-34662014000200007\&lng=en\&nrm=iso.

7. Rocha L, Herrera C, Vargas $\mathbf{O}$. Adherencia al tratamiento en pacientes en rehabilitación cardíaca: una propuesta de intervención. Tesis de grado. Bogotá: Universidad El Bosque Facultad de psicología; 2014.
8. La Rosa Y, Martín L, Bayarre H. Rev Cubana Med Gen Integr. [revista en Internet]. 2007 (Consultado marzo 2014); 23(1); Disponible en: http://scielo.sld. cu/scielo.php?script=sci_arttext\&pid=S0864-21252007000100005\&lng=es.

9. D'Anello S, Barreat Y, Escalante G, D'Orazio A, Benitez A. Relación médico paciente y adherencia a tratamiento. MedULA, [revista en Internet]. 2009; (Consultado marzo 2013);18(1):33-39. Disponible en: http://www.imbiomed.com.mx/1/1/ articulos.php?method=showDetail\&id_articulo=57110\&id_seccion=3485\&id_ ejemplar $=5778 \&$ id_revista $=147$

10. Guzmán Y, Estrada O, Tejada P, Crespo O. Factores relacionados con adherencia a un programa de rehabilitación. Revista Facultad de Salud Universidad Surcolombiana. 2010;2(1):39-50.

11. Delgado G, Figueroa C, Herrera C. Factores psicosociales asociados al compromiso con el tratamiento de pacientes de rehabilitación cardíaca. Tesis de grado de Especialización no publicada. Bogotá: Universidad El Bosque Facultad de psicología; 2012

12. Bandura A. Social cognitive theory of self-regulation. Organ Behav Hum Decis Process. 1991;(50):248-287

13. Karoly, P. Mechanisms of self-regulation: a view, Annu Rev Psychol. 1993;44:2352 .

14.Zimmerman B, Kitsantas A y Campillo M. Evaluación de la Autoeficacia Regulatoria: Una Perspectiva Social Cognitiva. Evaluar. 2005(5):1-21.

15. Tirapu J, García A, Luna P, Roig T, Pelegrin C. Modelos de funciones y control ejecutivo (I). Rev Neurol. 2008; 46 (11):684-692.

16. Amodio D, Master S, Yee C, Taylor S. Neurocognitive components of the behavioral inhibition and activation systems: Implications for theories of self-regulation. Psychophysiology. 2008 Jan;45(1):11-9

17. Verdejo-García A, Bechara A. Neuropsicología de las funciones ejecutivas Psicothema. 2010;22 (2):227-235

18. Levy B, Wagner A. Cognitive control and right ventrolateral prefrontal cortex: reflexive reorienting, motor inhibition, and action updating. Ann N Y Acad Sci. 2011 apr 12;1224(1):40-62

19. Gregory J, Beck J, Carr A. Goals, feedback, and self-regulation: Control theory as a natural framework for executive coaching. Consulting Psychology Journal: Practice and Research. 2011;63(1):26-38.

20. Sniehotta F, Scholz U, Schwarzer R. Bridging the intention-behaviour gap: planning, self-efficacy, and action control in the adoption and maintenance of physical exercise. Psychology and Health. 2005;20(2):143-160.

21. Sniehotta F, Scholz U, Schwarzer, R. Action plans and coping plans for physical exercise: A longitudinal intervention study in cardiac rehabilitation, $\mathrm{Br} J$ Health Psychol. 2006;11(1):23-27.

22. Labrador FJ, Cruzado JA, Muñoz M. Manual de técnicas de Modificación y terapia de conducta. Madrid: Ediciones Pirámide; 1993.

23. Rachlin H. Self-Control. Behaviorism. 1974;2 (1):94-107 
24.Pearson M, Kite B, Henson J. Predictive effects of good self-control and poor regulation on alcohol-related outcomes: do protective behavioral strategies mediate?. Psychol Addict Behav. (2013); 27(1):81-89.

25. Schwarzer R, Gutiérrez-Doña B. Modelando el cambio en el comportamiento de salud: Cómo predecir y modificar la adopción y el mantenimiento de comportamientos de salud. Revista Costarricense de Psicología. 2009;28(41-42): 11-39

26. Hall P, Fong G. Temporal self-regulation theory: A model for individual health behavior. Health Psychology Review. 2007; 1(1):6-52.

27. Hagger M, Wood Ch, Stiff Ch, Chatzisarantis L. The strength model of selfregulation failure and health-related behaviour. Health Psychology Review. 2009; 3(2):208-238.

28. Annesi J, Gorjala S. Relations of self-regulation and self-efficacy for exercise and eating and BMI change: A field investigation. Biopsychosoc Med.2010; 4(10).

29. Hall P, Fong G. Temporal self-regulation theory: a neurobiologically informed model for physical activity behavior. Front Hum Neurosci 2015; 9:1-8.

30. Flórez A L. Psicología social de la salud - Promoción y Prevención. Bogotá: El Manual Moderno; 2007.

31. Ortiz, M, Ortiz, E. Psicología de la salud: Una clave para comprender el fenómeno de la adherencia terapéutica. Rev Med Chil. 2007;135(5):647652. Consultado octubre 2013. Disponible en: http://www.scielo.cl/scielo. php?script=sci_arttext\&pid=S0034-98872007000500014\&lng=es. http://dx.doi. org/10.4067/S0034-98872007000500014.

32. Kyung $\mathbf{J}$. The effects of self-efficacy promoting cardiac rehabilitation program on self efficacy, health behavior, and quality of life. J Korean Acad Nurs 2003; 33(4):510-518

33. Janssen V, De Gucht V, van Exel H, Maes S. A self-regulation lifestyle program for post-cardiac rehabilitation patients has long-term effects on exercise adherence. J Behav Med. 2014 Apr;37(2):308-21. 